\title{
On finite groups all of whose cubic Cayley graphs are integral
}

\author{
Xuanlong Ma and Kaishun Wang \\ Sch. Math. Sci. \& Lab. Math. Com. Sys., \\ Beijing Normal University, 100875, Beijing, China.
}

\begin{abstract}
For any positive integer $k$, let $\mathcal{G}_{k}$ denote the set of finite groups $G$ such that all Cayley graphs $\operatorname{Cay}(G, S)$ are integral whenever $|S| \leq k$. Estélyi and Kovács [14] classified $\mathcal{G}_{k}$ for each $k \geq 4$. In this paper, we characterize the finite groups each of whose cubic Cayley graphs is integral. Moreover, the class $\mathcal{G}_{3}$ is characterized. As an application, the classification of $\mathcal{G}_{k}$ is obtained again, where $k \geq 4$.
\end{abstract}

Keywords: Cayley graph, integral graph, Cayley integral group, eigenvalue. MSC 2010: 05C25, 05C50, 20C10.

\section{Introduction}

A graph is integral if all its eigenvalues are integers. Harary and Schwenk [16] introduced integral graphs, and proposed the problem of classifying integral graphs. Since then classifications of some special integral graphs have received considerable attention, see $[2,7,9,11,12,20]$. For more information, see the two surveys $[4,8]$.

Let $G$ be a finite group. A subset $S$ of $G$ is called symmetric if $S^{-1}=S$. If $S$ is a symmetric subset of $G$ and does not contain the identity, then the Cayley graph $\operatorname{Cay}(G, S)$ is the graph with vertex set $G$ and edge set $\{\{g, s g\}: g \in G, s \in S\}$. Abdollahi and Vatandoost [3] listed some infinite families of integral Cayley graphs, and classified connected cubic integral Cayley graphs.

A finite group is a Cayley integral group if each of its Cayley graphs is integral. Klotz and Sander [17] introduced this concept and determined all abelian Cayley

E-mail addresses: xuanlma@mail.bnu.edu.cn (X. Ma), wangks@bnu.edu.cn (K. Wang). 
integral groups. The nonabelian case was handled by Abdollahi and Jazaeri [1], and independently by Ahmady et al. [5].

Theorem 1.1. ([17, Theorem 13], [1, Theorem 1.1], [5, Theorem 4.2.]) All finite Cayley integral groups are

$$
\mathbb{Z}_{2}^{m} \times \mathbb{Z}_{3}^{n}, \mathbb{Z}_{2}^{m} \times \mathbb{Z}_{4}^{n}, S_{3}, Q_{8} \times \mathbb{Z}_{2}^{n}, \operatorname{Dic}\left(\mathbb{Z}_{6}\right)
$$

where $m, n \geq 0, Q_{8}$ is the quaternion group of order 8 and $\operatorname{Dic}\left(\mathbb{Z}_{6}\right)$ is the generalized dicyclic group of order 12 .

Very recently, Estélyi and Kovács [14] generalized this class of groups by introducing the class $\mathcal{G}_{k}$ of finite groups $G$ such that all Cayley graphs Cay $(G, S)$ are integral whenever $|S| \leq k$, and they classified $\mathcal{G}_{k}$ for each $k \geq 4$.

Theorem 1.2. ([14, Theorem 1.3]) Every class $\mathcal{G}_{k}$ consists of the Cayley integral groups if $k \geq 6$. Moreover, $\mathcal{G}_{4}$ and $\mathcal{G}_{5}$ are equal, and consist of the Cayley integral groups and $\operatorname{Dic}\left(\mathbb{Z}_{3}^{n} \times \mathbb{Z}_{6}\right)$, where $n \geq 1$.

For any positive integer $k \geq 2$, let $\mathcal{A}_{k}$ denote the set of finite groups any of whose Cayley graphs with valency $k$ is integral. We note that If $G$ is group of odd order, then $G \notin \mathcal{A}_{m}$ for each odd integer $m$.

In this paper we focus on the study of $\mathcal{A}_{3}$. In Section 2 , we characterize $\mathcal{A}_{3}$, and show that $\mathcal{G}_{3}$ consists of $\mathcal{A}_{3}$ and all finite 3 -groups of exponent 3 . As an application, we give an alternating proof Theorem 1.2 in Section 3.

\section{Classes $\mathcal{A}_{3}$ and $\mathcal{G}_{3}$}

Denote by $D_{n}$ the dihedral group of order $n$. Let $V$ be a vector space over complex field $\mathbb{C}$. A representation of group $G$ on $V$ is a group homomorphism $\rho$ from $G$ to $G L(V)$, the group of invertible linear maps from $V$ to itself. A subspace $W$ of $V$ is said to be invariant under $\rho$ provided that $w^{g^{\rho}} \in W$ for any $g \in G$ and $w \in W$. If $V$ has no nontrivial $\rho$-invariant subspaces, then $\rho$ is called an irreducible representation of $G$.

Proposition 2.1. ([13, Theorem 3]) Given a Cayley graph $\operatorname{Cay}(G, S)$, let

$$
\left\{\rho_{1}, \rho_{2}, \ldots, \rho_{t}\right\}
$$

be the set of all irreducible representations of $G$. Then $\bigcup_{i=1}^{t} \Omega_{i}$ is the set of all eigenvalues of $\operatorname{Cay}(G, S)$, where $\Omega_{i}$ is the set of all eigenvalues of the matrix

$$
\rho_{i}(S)=\sum_{s \in S} \rho_{i}(s) .
$$


Proposition 2.2. ([3, Theorem 1.1]) A cubic connected Cayley graph Cay $(G, S)$ is integral if and only if $G$ is isomorphic one the following groups:

$$
\mathbb{Z}_{2}^{2}, \mathbb{Z}_{4}, \mathbb{Z}_{6}, \mathbb{Z}_{2}^{3}, \mathbb{Z}_{2} \times \mathbb{Z}_{4}, \mathbb{Z}_{2} \times \mathbb{Z}_{6}, S_{3}, D_{8}, D_{12}, A_{4}, S_{4}, D_{8} \times \mathbb{Z}_{3}, D_{6} \times \mathbb{Z}_{4}, A_{4} \times \mathbb{Z}_{2}
$$

Let $G \in \mathcal{A}_{k}$ and $H$ be a subgroup of $G$. If $H$ has a subset $S$ such that $|S|=k$ and $S=S^{-1}$, since $\operatorname{Cay}(G, S)$ is a disjoint union of some Cay $(H, S)$, one has that Cay $(H, S)$ is integral. In particular, if $K \in \mathcal{G}_{k}$, then every subgroup of $K$ belongs to $\mathcal{G}_{k}$. Denote by $\mathcal{G}$ the set of all finite groups $G$ with $\{|g|: g \in G\} \subseteq\{1,2,3,4,6\}$.

Lemma 2.3. A finite group $G$ belongs to $\mathcal{A}_{2}$ if and only if $G \in \mathcal{G}$, and $G$ is $D_{8}$-free and $D_{12}$-free.

Proof. Suppose that $G \in \mathcal{A}_{2}$. It is well known that the cycle of length $n$ is integral if and only if $n=3,4$ or 6 (cf. [10, p. 9]). This implies that $G \in \mathcal{G}$. Furthermore, if $a$ and $b$ are two generators of $D_{8}$ such that $|a|=|b|=2$, then $\operatorname{Cay}\left(D_{8},\{a, b\}\right)$ is the cycle of length 8 and so $D_{8} \notin \mathcal{A}_{2}$. Similarly, we have $D_{12} \notin \mathcal{G}_{2}$. It follows that $G$ is $D_{8}$-free and $D_{12}$-free.

For the converse, let $\operatorname{Cay}(G, S)$ with valency 2 . Then $S=\{x, y\}$ or $S=\left\{z, z^{-1}\right\}$, where $x$ and $y$ are two distinct involutions, and $z$ is of order greater than 2 . For the former, one has that $\langle x, y\rangle \cong \mathbb{Z}_{2}^{2}$ or $D_{6}$, since $\mathbb{Z}_{2}^{2}$ and $D_{6}$ all are Cayley integral, it follows that $\operatorname{Cay}(G,\{x, y\})$ is integral. For the latter, obvious that $\operatorname{Cay}\left(G,\left\{z, z^{-1}\right\}\right)$ is integral. Thus, we have $G \in \mathcal{A}_{2}$.

Lemma 2.4. The alternating group $A_{4}$ belongs to $\mathcal{A}_{3}$.

Proof. Suppose that $\operatorname{Cay}\left(A_{4}, S\right)$ is a Cayley graph with valency 3 .

Case 1. $S$ consists of three involutions.

It is clear that $\langle S\rangle \cong \mathbb{Z}_{2}^{2}$. Thereby, we have that $\operatorname{Cay}(\langle S\rangle, S)$ is integral and so is $\operatorname{Cay}\left(A_{4}, S\right)$.

Case 2. $S$ consists of an involution and two elements of order 3.

For $i=1$ or 2 , suppose that $T_{i}=\left\{x_{i}, y_{i}, y_{i}^{-1}\right\}$, where $x_{i}, y_{i} \in A_{4},\left|x_{i}\right|=2,\left|y_{i}\right|=3$, and. Then $\left\langle T_{i}\right\rangle=A_{4}$, and it is easy to check that the mapping $\sigma: x_{1} \longmapsto x_{2}, y_{1} \longmapsto y_{2}$ is an automorphism of $A_{4}$. This means that $\sigma$ is an isomorphism from $\operatorname{Cay}\left(A_{4}, T_{1}\right)$ to $\operatorname{Cay}\left(A_{4}, T_{2}\right)$. Consequently, to see $A_{4} \in \mathcal{A}_{3}$, it is sufficient to prove that $\operatorname{Cay}\left(A_{4}, T_{1}\right)$ is integral. Take $T_{1}=S=\{(1,3)(2,4),(2,4,3),(2,3,4)\}$ and let $\omega=e^{\frac{2 \pi}{3} i}$. By GAP [15] all nontrivial irreducible representations of $A_{4}$ are given by

$$
\rho_{1}:(2,4,3) \mapsto \omega,(1,3)(2,4) \mapsto 1 ; \rho_{2}:(2,4,3) \mapsto \omega^{2},(1,3)(2,4) \mapsto 1 ;
$$




$$
\rho_{3}:(2,4,3) \longmapsto\left(\begin{array}{lll}
0 & 0 & 1 \\
1 & 0 & 0 \\
0 & 1 & 0
\end{array}\right),(1,3)(2,4) \longmapsto\left(\begin{array}{ccc}
-1 & 0 & 0 \\
0 & 1 & 0 \\
0 & 0 & -1
\end{array}\right) .
$$

By Proposition 2.1, it is easy to check that $\operatorname{Cay}\left(A_{4}, S\right)$ is integral.

Proposition 2.5. Let $G \in \mathcal{A}_{3}$. Then $G$ has a subgroup isomorphic to $S_{3}$ if and only if $G \cong S_{3}$.

Proof. Assume that $K$ is a subgroup of $G$ isomorphic to $S_{3}$. Let $\{x, y, z\}$ be the set of all involutions of $K$.

Suppose that $a$ is an involution in $G \backslash\{x, y\}$. Then $\langle x, y, a\rangle$ is nonabelian and Cay $(\langle x, y, a\rangle,\{x, y, a\})$ is a cubic connected integral graph. Note that $\langle x, y\rangle \cong S_{3}$. It follows from Proposition 2.2 that $\langle x, y, a\rangle$ is one the following groups:

$$
S_{3}, D_{12}, S_{4}, D_{6} \times \mathbb{Z}_{4} .
$$

We now claim $D_{8} \notin \mathcal{A}_{3}$. Let $D_{8}=\left\langle a, b: a^{4}=b^{2}=1, b a b=a^{3}\right\rangle$. Then by GAP [15], $D_{8}$ has a 2-dimensional irreducible representation $\rho_{1}$ given by

$$
a \longmapsto\left(\begin{array}{cc}
0 & -1 \\
1 & 0
\end{array}\right), b \longmapsto\left(\begin{array}{cc}
0 & 1 \\
1 & 0
\end{array}\right) .
$$

Set $T_{1}=\left\{a^{2}, a^{3} b, b\right\}$. Then we have

$$
\sum_{s \in T_{1}} \rho_{1}(s)=\left(\begin{array}{cc}
0 & 1 \\
1 & -2
\end{array}\right)
$$

which implies that $-1 \pm \sqrt{2}$ is an eigenvalue of $\operatorname{Cay}\left(D_{8}, T_{1}\right)$ by Proposition 2.1. Thus, $D_{8} \notin \mathcal{A}_{3}$.

Now we prove that $D_{12} \notin \mathcal{A}_{3}$. Let $D_{12}=\left\langle a, b: a^{6}=b^{2}=1, b a b=a^{3}\right\rangle$. Then $D_{12}$ has a 2-dimensional irreducible representation $\rho_{2}$ determined by

$$
a^{5} b \longmapsto\left(\begin{array}{cc}
0 & \omega \\
\omega^{2} & 0
\end{array}\right), b \longmapsto\left(\begin{array}{cc}
0 & -1 \\
-1 & 0
\end{array}\right)
$$

Take $T_{2}=\left\{a^{3}, a^{5} b, b\right\}$. It follows that

$$
\sum_{s \in T_{2}} \rho_{2}(s)=\left(\begin{array}{cc}
-1 & \omega-1 \\
\omega^{2}-1 & -1
\end{array}\right)
$$

which has eigenvalues $-1 \pm \sqrt{3}$ and so one has that $D_{12} \notin \mathcal{A}_{3}$. 
Note that $S_{4}$ contains a subgroup isomorphic to $D_{8}$, and $D_{6} \times \mathbb{Z}_{4}$ has a subgroup isomorphic to $D_{12}$. Since $\langle x, y, a\rangle$ belongs to $\mathcal{A}_{3}$, by (1) one has that $\langle x, y, a\rangle \cong S_{3}$. This means that $a=z$. Thus, $G$ has precisely three distinct involutions and so $K$ is normal in $G$. Note that if $G$ has an element $g$ of order greater than 2, then Cay $\left(\left\langle x, g, g^{-1}\right\rangle,\left\{x, g, g^{-1}\right\}\right)$ is a cubic connected integral graph. By Proposition 2.2, it is easy to see that $G \in \mathcal{G}$.

Suppose that $b$ is an element of $G$ with $|b|=4$. Let $g$ be an arbitrary involution of $G$. If $\langle g, b\rangle$ is nonanelian, then $\langle g, b\rangle \cong A_{4}$ or $S_{3}$, a contradiction since $A_{4}$ and $S_{3}$ have no elements of order 4 . Hence, one gets $[g, b]=1$, where $[g, b]$ is the commutator of $g$ and $b$, that is, $[g, b]=g^{-1} b^{-1} g b$. It follows that $\left[b^{2}, g\right]=1$, which is impossible. Thus, $G$ has no elements of order 4 . By a similar argument, $G$ also has no elements of order 6 . Thereby, it follows that $\{|g|: g \in G\} \subseteq\{1,2,3\}$.

Let $w$ belong to the centralizer of $K$ in $G$. If $|w|=3$ then $G$ has an element $w x$ with order 6 , a contradiction. Furthermore, since all involutions are pairwise noncommutative, one has $|w| \neq 2$. This means that $C_{G}(K)=1$. By the $N / C$ Theorem (cf. [21, Theorem 1.6.13]), we obtain that $G$ is isomorphic to a subgroup of the full automorphism group $\operatorname{Aut}(K)$ of $K$. Note that $\operatorname{Aut}(K) \cong S_{3}$. Thus, we conclude that $G \cong S_{3}$.

The converse implication is straightforward.

By the definition of $\mathcal{A}_{3}$, we see that every group of odd order does not belong to $\mathcal{A}_{3}$. Now we give a characterization for class $\mathcal{A}_{3}$.

Theorem 2.6. Let $G$ be a finite group. Then $G \in \mathcal{A}_{3}$ if and only if $G \cong S_{3}$, or for any involution $x$ and element $y \in G,\langle x, y\rangle$ is isomorphic to one of the following groups:

$$
\mathbb{Z}_{2}, \mathbb{Z}_{2}^{2}, \mathbb{Z}_{4}, \mathbb{Z}_{6}, \mathbb{Z}_{2} \times \mathbb{Z}_{4}, \mathbb{Z}_{2} \times \mathbb{Z}_{6}, A_{4} .
$$

Proof. " $\Rightarrow "$ : Case 1. $|y|=1$.

It is clear that $\langle x, y\rangle \cong \mathbb{Z}_{2}$.

Case 2. $|y|=2$.

If $y=x$ or $[x, y]=1$, then $\langle x, y\rangle \cong \mathbb{Z}_{2}$ or $\mathbb{Z}_{2}^{2}$, as desired. Note that two distinct involutions generate a dihedral group. Thus, we may suppose that $\langle x, y\rangle \cong D_{2 n}$, where $n \geq 3$. This implies that $\operatorname{Cay}(\langle x, y\rangle,\{x, y, z\})$ is integral, where $z$ is an involution of $\langle x, y\rangle \backslash\{x, y\}$. Since $D_{8}, D_{12} \notin \mathcal{A}_{3}$, one has that $\langle x, y\rangle \cong S_{3}$ by Proposition 2.2.

Case 3. $|y| \geq 3$.

Then $\operatorname{Cay}\left(\left\langle x, y, y^{-1}\right\rangle,\left\{x, y, y^{-1}\right\}\right)$ is a cubic connected integral graph. Note that $S_{4}, D_{8} \times \mathbb{Z}_{3}, D_{6} \times \mathbb{Z}_{4}, A_{4} \times \mathbb{Z}_{2} \notin \mathcal{A}_{3}$. By Proposition 2.2 again, one has that $\langle x, y\rangle \cong$ $\mathbb{Z}_{4}, \mathbb{Z}_{6}, \mathbb{Z}_{2} \times \mathbb{Z}_{4}, \mathbb{Z}_{2} \times \mathbb{Z}_{6}, S_{3}$ or $A_{4}$.

From the above, now the desired result follows from Proposition 2.5. 
" $\Leftarrow "$ ": It is clear that $S_{3} \in \mathcal{A}_{3}$. We may assume that $G ¥ S_{3}$. Let $S$ be an arbitrary symmetric subset of $G$ with $|S|=3$ and $1 \notin S$.

Case 1. $S$ consists of three involutions.

Note that every two elements of $S$ commute. So $\langle S\rangle \cong \mathbb{Z}_{2}^{2}$ or $\mathbb{Z}_{2}^{3}$. This means that $\operatorname{Cay}(G, S)$ is integral.

Case 2. $S=\left\{x, y, y^{-1}\right\}$ with $|x|=2$ and $|y| \geq 3$.

If $[x, y] \neq 1$, then $\langle x, y\rangle \cong A_{4}$, by Lemma 2.4 one concludes that Cay $(G, S)$ is integral. If $\langle x, y\rangle$ is abelian, then $\langle x, y\rangle$ is Cayley integral and so is Cay $(G, S)$.

Note that all 3-groups of exponent 3 are contained in $\mathcal{G}_{3}$, but they are not contain in $\mathcal{A}_{3}$. Combining Lemma 2.3 and Theorem 2.6, we obtain a characterization of $\mathcal{G}_{3}$.

Corollary 2.7. The class $\mathcal{G}_{3}$ consists of all finite 3-groups of exponent 3 and all finite groups in $\mathcal{A}_{3}$.

Corollary 2.8. Let $G$ be a nilpotent group. Then $G \in \mathcal{G}_{3}$ if and only if one of the following holds:

(1) $G$ is a 3-group of exponent 3;

(2) $G \cong \mathbb{Z}_{2}^{n}$ for $n \geq 1$;

(3) $G$ is a 2-group of exponent 4, and every involution of $G$ belongs to $Z(G)$, the center of $G$;

(4) $G \cong \mathbb{Z}_{2}^{n} \times B$, where $B$ is an arbitrary 3-group with exponent 3 , and $n \geq 1$.

As pointed out in [18], $\mathcal{G}_{3}$ is much wider than the classes $\mathcal{G}_{k}$ for $k \geq 4$. We now present some examples belonging to $\mathcal{G}_{3}$, however, they all do not belong to $\mathcal{G}_{4}$. Firstly, by Corollary 2.7 we see that $A_{4} \in \mathcal{G}_{3}$.

Example 2.9. Let $A$ be an abelian group with exponent 4. Then $Q_{8} \times A \in \mathcal{G}_{3}$. It is because that for any involution $t$ of $Q_{8} \times A$, we have that $t=(-1,1),(1, x)$ or $(-1, x)$, where $x$ is an involution of $A$. Thereby, $t \in Z\left(Q_{8}\right) \times A=Z\left(Q_{8} \times A\right)$. In view of Corollary 2.8, one has that $Q_{8} \times A \in \mathcal{G}_{3}$.

Example 2.10. Let $B$ be a group with exponent 3 and let $A_{4} \backslash\{(1)\}=T \cup H$, where $T$ is the set of all involutions and $H$ is the set of all elements of order 3 . Note that every involution in $A_{4} \times B$ has the form $(t, 1)$ for some $t \in T$. It is easy to see that if $t_{0}, t \in T$ and $h \in H$, then $\left[(t, 1),\left(t_{0}, 1\right)\right]=1,\left[(t, 1),\left(t_{0}, a\right)\right]=1$ and $\langle(t, 1),(h, 1)\rangle \cong A_{4}$, where $1 \neq a \in B$. Now take $t \in T$ and $h \in H$, one has that

$$
\left\langle(t, 1),(h, a):(t, 1)^{2}=(h, a)^{3}=1,((h, a)(t, 1))^{3}=1\right\rangle \cong A_{4} .
$$

Thus, we have that $A_{4} \times B \in \mathcal{G}_{3}$ by Corollary 2.7. 
Example 2.11. By Corollary 2.7, if $G \in \mathcal{G}$ and every involution of $G$ is central, then $G \in \mathcal{G}_{3}$. For example, the special linear group $S L(2,3)$, clearly, $\{|g|: g \in S L(2,3)\}=$ $\{1,2,3,4,6\}$ and $S L(2,3)$ has precisely one involution, so $G \in \mathcal{G}_{3}$. Particularly, $\mathbb{Z}_{2}^{n} \times S L(2,3) \in \mathcal{G}_{3}$ for each $n \geq 2$.

\section{Proof of Theorem 1.2}

In this section, by using Corollary 2.7, we give an alternative proof of Theorem 1.2. By [14, Lemma 2.4], for each $n \geq 1$, the groups $\operatorname{Dic}\left(\mathbb{Z}_{3}^{n} \times \mathbb{Z}_{6}\right)$ belongs to $\mathcal{G}_{5} \backslash \mathcal{G}_{6}$. In order to prove Theorem 1.2 , we only need to show that any group in $\mathcal{G}_{4}$ is a Cayley integral group or $\operatorname{Dic}\left(\mathbb{Z}_{3}^{n} \times \mathbb{Z}_{6}\right)$, where $n$ is a positive integer.

Lemma 3.1. Let $G$ be a nilpotent group. If $G \in \mathcal{G}_{4}$, then $G$ is Cayley integral.

Proof. Note that $\mathcal{G}_{4} \subseteq \mathcal{G}_{2}$. Then, by Lemma 2.3 we have that $G \in \mathcal{G}$. It means that $|G|$ has at most two distinct prime divisor 2 and 3 .

Case 1. $G$ is a 3 -group.

If $G$ is abelian, then $G$ is elementary abelian and so $G$ is Cayley integral, as desired.

Suppose that $G$ is nonabelian. Since $G$ is of exponent $3, G$ has two elements $a, b$ such that $|a|=|b|=3$ and $[a, b] \neq 1$. It follows that $\langle a, b\rangle$ is the nonabelian group of order 27 and exponent 3, where $\langle a, b\rangle=\left\langle a, b: a^{3}=b^{3}=(a b)^{3}=\left(a b^{2}\right)^{3}=1\right\rangle$. Take $S=\left\{a, a^{2}, b, b^{2}\right\}$. Note that $\langle a, b\rangle$ has a 3 -dimensional irreducible representation given by

$$
a \longmapsto\left(\begin{array}{ccc}
1 & 0 & 0 \\
0 & \omega & 0 \\
0 & 0 & \omega^{2}
\end{array}\right), b \longmapsto\left(\begin{array}{ccc}
0 & 0 & \omega^{2} \\
\omega & 0 & 0 \\
0 & 1 & 0
\end{array}\right)
$$

It is easy to check that $\operatorname{Cay}(\langle a, b\rangle, S)$ is not integral, which is a contradiction as $\langle a, b\rangle \in \mathcal{G}_{4}$.

Case 2. $G$ is a 2-group.

It is clear that $G$ is Cayley integral if $G$ is abelian. Thus, we may assume that $G$ is nonabelian. Then $G$ is of exponent 4 and by Corollary 2.8, one has that every cyclic subgroup of order 2 is central in $G$.

Now we show that every cyclic subgroup of order 4 is also normal in $G$. To see this, suppose, to the contrary, that there exist two elements $x$ and $y$ in $G$ such that $|x|=4$ and $x^{y} \notin\langle x\rangle$. Then $|y|=4$ and $[x, y] \neq 1$. Considering

$$
H=\left\langle x, y: x^{4}=y^{4}=\left[x^{2}, y\right]=\left[x, y^{2}\right]=(x y)^{4}=1\right\rangle,
$$


with the help of GAP [15], one concludes that $H \cong H_{0}, H_{1}, H_{2}$ or $Q_{8}$, where

$$
\begin{gathered}
H_{0}=\left\langle a, b, c: a^{4}=b^{4}=c^{2}=1,[a, b]=c,[c, a]=[c, b]=1\right\rangle, \\
H_{1}=\left\langle a_{1}, b_{1}: a_{1}^{4}=b_{1}^{4}=1, a_{1}^{b_{1}}=a_{1}^{-1}\right\rangle, \\
H_{2}=\left\langle a_{2}, b_{2}, c_{2}: a_{2}^{4}=b_{2}^{2}=c_{2}^{2}=1,\left[a_{2}, b_{2}\right]=c_{2},\left[c_{2}, a_{2}\right]=\left[c_{2}, b_{2}\right]=1\right\rangle .
\end{gathered}
$$

Now we prove that $H_{0}, H_{1}$ and $H_{2}$ do not belong to $\mathcal{G}_{4}$. Note that $b_{2}$ is an involution of $H_{2}$, and $b_{2} \notin Z\left(H_{2}\right)$. Consequently, we have that $H_{2} \notin \mathcal{G}_{3}$ by Corollary 2.8, and hence $H_{2} \notin \mathcal{G}_{4}$. For $H_{1}$, set $T_{1}=\left\{a_{1}^{2} b_{1}^{-1}, b_{1} a_{1}^{2}, a_{1}^{-1} b_{1}^{-1}, b_{1} a_{1}\right\}$, since $H_{1}$ has a 2-dimensional irreducible representation given by

$$
a_{1} \longmapsto\left(\begin{array}{cc}
0 & -1 \\
1 & 0
\end{array}\right), b_{1} \longmapsto\left(\begin{array}{ll}
0 & 1 \\
1 & 0
\end{array}\right)
$$

it is easy to check that $\operatorname{Cay}\left(H_{1}, T_{1}\right)$ is not integral. For $H_{0}$, by verifying one see that $H_{0}=\left\langle b a^{2}, a^{3} b^{2}\right\rangle$, take $T_{0}=\left\{b a^{2}, a^{2} b^{3}, a^{3} b^{2}, b^{2} a\right\}$. Note that $H_{0}$ has a 2-dimensional irreducible representation given by

$$
b a^{2} \longmapsto\left(\begin{array}{cc}
0 & 1 \\
1 & 0
\end{array}\right), a^{3} b^{2} \longmapsto\left(\begin{array}{cc}
1 & 0 \\
0 & -1
\end{array}\right)
$$

This implies that $\operatorname{Cay}\left(H_{0}, T_{0}\right)$ is not integral.

Considering the above we see that $H \cong Q_{8}$, which is a contradiction since every subgroup of $Q_{8}$ is normal. This yields that every cyclic subgroup of order 4 is normal in $G$.

Now note that every cyclic subgroup of $G$ is normal and so is every subgroup of $G$. Consequently, $G$ is isomorphic to a direct product of $Q_{8}$, an elementary abelian 2-group and an abelian group of odd order (cf. [6]), one gets that $G \cong Q_{8} \times \mathbb{Z}_{2}^{m}$ for some nonnegative integer $m$. By Theorem 1.1 one has that $G$ is Cayley integral, as desired.

Case 3. $G=P \times Q$, where $P$ and $Q$ are the Sylow 2- and 3-subgroups of $G$, respectively.

By Case 1, one has that $Q$ is elementary abelian. Since $G$ has no elements of order 12 , one gets that $P$ is also elementary abelian. It means that $G$ is abelian, and so $G$ is Cayley integral.

Lemma 3.2. Let $G$ be a nonnilpotent group. If $G \in \mathcal{G}_{4}$, then $G \cong S_{3}$ or $G \cong$ $\operatorname{Dic}\left(\mathbb{Z}_{3}^{n} \times \mathbb{Z}_{6}\right)$, where $n$ is a nonnegative integer. 
Proof. We first claim that $A_{4} \notin \mathcal{G}_{4}$. Clearly, $A_{4}=\langle(2,4,3),(1,3)(2,4),(1,2)(3,4)\rangle$. Take $S=\{(2,3,4),(2,4,3),(1,3)(2,4),(1,2)(3,4)\}$. Note that $A_{4}$ has a 3 -dimensional irreducible representation determined by

$$
\begin{aligned}
(2,4,3) \longmapsto & \left(\begin{array}{lll}
0 & 0 & 1 \\
1 & 0 & 0 \\
0 & 1 & 0
\end{array}\right),(1,3)(2,4) \longmapsto\left(\begin{array}{ccc}
-1 & 0 & 0 \\
0 & 1 & 0 \\
0 & 0 & -1
\end{array}\right), \\
& (1,2)(3,4) \longmapsto\left(\begin{array}{ccc}
1 & 0 & 0 \\
0 & -1 & 0 \\
0 & 0 & -1
\end{array}\right)
\end{aligned}
$$

By verifying one has that $\operatorname{Cay}\left(A_{4}, S\right)$ is not integral, so our claim is valid.

Note that $G \in \mathcal{G}_{3}$. By Corollary 2.7 and Proposition 2.5, we may assume that $G \nsubseteq S_{3}$. Then again by Corollary 2.7 and $A_{4} \notin \mathcal{G}_{4}$, every involution of $G$ belongs to $Z(G)$. Since $G$ is not nilpotent, $G$ has elements of order 4 . Suppose that $b$ and $a$ are two elements of $G$ such that $|b|=4$ and $|a|=3$. Note that $G$ has no elements of order 12. Then $[a, b] \neq 1$. Since $G \in \mathcal{G}_{4}$, $\operatorname{Cay}\left(\langle a, b\rangle,\left\{a, a^{-1}, b, b^{-1}\right\}\right)$ is a quartic connected integral graph. Note that all quartic connected Cayley integral graphs were obtained in [19]. Observe that $a^{b}=a^{-1}$.

Now we claim that $G$ has a unique involution. Suppose, to the contrary, that there exists an involution $u$ in $G$ such that $u \neq b^{2}$. Then $G$ has a subgroup

$$
H=\left\langle a, b, u: a^{3}=b^{4}=u^{2}=1,[a, u]=[b, u]=1, a^{b}=a^{-1}\right\rangle \cong\left(\mathbb{Z}_{3} \rtimes \mathbb{Z}_{4}\right) \times \mathbb{Z}_{2} .
$$

Let $S=\left\{b^{-1} u, u b, b a, a^{-1} b^{-1}\right\}$. Note that there exists a irreducible representation of $H$ given by

$$
a^{-1} \longmapsto\left(\begin{array}{cc}
\omega & 0 \\
0 & \omega^{2}
\end{array}\right), b \longmapsto\left(\begin{array}{cc}
0 & -1 \\
-1 & 0
\end{array}\right), u \longmapsto\left(\begin{array}{cc}
-1 & 0 \\
0 & -1
\end{array}\right) .
$$

It is easy to check that $\operatorname{Cay}(H, S)$ is not integral, contrary to $H \in \mathcal{A}_{4}$. This forces that the claim is valid and thereby, $G$ has a Sylow 2-subgroup $P$ that is isomorphic to $\mathbb{Z}_{4}$ or $Q_{8}$; this is because that $P$ has a unique subgroup of order 2 (cf. [22, pp. 252, Theorem 9.7.3]). Write

$$
Q_{8}=\left\{ \pm 1, \pm i, \pm j, \pm k: i^{2}=j^{2}=k^{2}=-1, i j=k=-j i\right\} .
$$

Note that $|a|=3$. If $P=Q_{8}$ then $a^{i j}=a^{-1}=\left(a^{-1}\right)^{j}$ and so $\left|a^{-1} j\right|=12$, a contradiction. It follows that $P=\langle b\rangle$. Note that $G$ has a subgroup $Q$ isomorphic to $\mathbb{Z}_{3}^{m} \times \mathbb{Z}_{2}$ for $m \geq 1$, and for each $x \in Q$ one has $x^{b}=x^{-1}$. Thus, $G$ is isomorphic to $\operatorname{Dic}\left(\mathbb{Z}_{3}^{n} \times \mathbb{Z}_{6}\right)$ for $n \geq 0$.

Combining Lemma 3.1 and Lemma 3.2, we complete the proof of Theorem 1.2. 


\section{Acknowledgement}

The authors are grateful to the referee for a very careful reading of the paper, and many useful suggestions and comments. This research is supported by National Natural Science Foundation of China $(11271047,11371204)$ and the Fundamental Research Funds for the Central University of China.

\section{References}

[1] A. Abdollahi and M. Jazaeri, Groups all of whose undirected Cayley graphs are integral, European J. Combin. 38 (2014) 102-109.

[2] A. Abdollahi and M. Jazaeri, On groups admitting no integral Cayley graphs besides complete multipartite graphs, Appl. Anal. Discrete Math. 7 (2013) 119128.

[3] A. Abdollahi and E. Vatandoost, Which Cayley graphs are integral? Electron. J. Combin. 16 (2009) \#R122.

[4] O. Ahmadi, N. Alon, L.F. Blake and I.E. Shparlinski, Graphs with integral spectrum, Linear Algebra Appl. 430 (2009) 547-552.

[5] A. Ahmady, J.P. Bell and B. Mohar, Integral Cayley graphs and groups, SIAM J. Discrete Math. 28 (2014) 685-701.

[6] R. Baer, Situation der Untergruppen und Struktur der Gruppe, S.B. Heidelberg Akad. Wiss. 2 (1933), 12-17.

[7] K. Balińska, D. Cvetković, M. Lepović and S. Simić, There are exactly 150 connected integral graphs up to 10 vertices, Univ. Beograd Publ. Elektrotehn. Fak. Ser. Mat. 10 (1999) 95-105.

[8] K. Balińska, D. Cvetković, Z. Radosavljević, S. Simić and D. Stevanović, A survey on integral graphs, Univ. Beograd Publ. Elektrotehn. Fak. Ser. Mat. 13 (2002) 42-65.

[9] K. Balińska, S.K. Simić, T. Zwierzyński and T. Krzysztof, Which non-regular bipartite integral graphs with maximum degree four do not have \pm 1 as eigenvalues? Discrete Math. 286 (2004) 15-24.

[10] A.E. Brouwer, W.H. Haemers, Spectra of graphs, Springer-Verlag, New York, 2012 . 
[11] F.C. Bussemaker, D. Cvetković, There are exactly 13 connected, cubic, integral graphs, Univ. Beograd Publ. Elektrotehn. Fak. Ser. Mat. Fiz. 544-576 (1976) 43-48.

[12] T. Chung, J. Koolen, Y. Sano and T. Taniguchi, The non-bipartite integral graphs with spectral radius three, Linear Algebra Appl. 435 (2011) 2544-2559.

[13] P. Diaconis and M. Shahshahani, Generating a random permutation with random transpositions, Z. Wahsch. Verw. Gebiete 57 (1981) 159-179.

[14] I. Estélyi and I. Kovács, On groups all of whose undirected Cayley graphs of bounded valency are integral, Electron. J. Combin. 21 (2014) \#P4.45.

[15] GAP - Groups, Algorithms, Programming - a System for Computational Discrete Algebra, Version 4.6.5, 2013, http://gap-system.org.

[16] F. Harary and A.J. Schwenk, Which Graphs Have Integral Spectra? in: Lecture Notes in Mathematics, 406, Springer, 1974, pp. 45-51.

[17] W. Klotz and T. Sander, Integral Cayley graphs over abelian groups, Electron. J. Combin. 17 (2010) \#R81.

[18] I. Kovács, On groups all of whose undirected Cayley graphs of bounded valency are integral, Modern Trends in Algebraic Graph Theory, Villanova University, June 2-5, 2014, http://www.csc.villanova.edu/ModernTrends/MSC154/ Day3Talks/IstvanKovacs . pdf.

[19] M. Minchenko and I.M. Wanless, Quartic integral Cayley graphs, Ars Math. Contemp. 8 (2015) 381-408.

[20] G.R. Omidi, On integral graphs with few cycles, Graphs Combin. 25 (2009) 841-849.

[21] D.J. Robinson, A Course in the Theory of Group, Springer-Verlag, New York, 1996.

[22] W.R. Scott, Group theory, Prentice-Hall, New Jersey, 1964. 